\title{
MORPHOLOGICAL HIT-OR-MISS TRANSFORM BASED APPROACH FOR BUILDING DAMAGE ESTIMATION FROM VHR AIRBORNE IMAGERY IN 2011 PACIFIC COAST OF TOHOKU EARTHQUAKE AND TSUNAMI
}

\author{
Chandana Dinesh Kumara Parape ${ }^{\mathrm{a},}$, H. Chinthaka N. Premachandra ${ }^{\mathrm{b}}$, Masayuki Tamura ${ }^{\mathrm{c}}$, Masami Sugiura $^{\mathrm{d}}$ \\ ${ }^{a}$ Department of Urban and Environmental Engineering, \\ Graduate School of Engineering, Kyoto University, Japan \\ ${ }^{\mathrm{b}}$ Department of Electrical Engineering, Tokyo University of Science, Tokyo, Japan \\ ${ }^{\mathrm{c}}$ Graduate School of Global Environmental Studies, \\ Kyoto University, Japan \\ ${ }^{\mathrm{d}}$ Asia Disaster Reduction Center, Kobe, Japan \\ Commission ICWG III/VII
}

KEY WORDS: Mathematical Morphological Operators, Hit-or-Miss Transform, Natural Hazard, VHR Airborne Images, Building Extraction.

\begin{abstract}
:
The very high resolution (VHR) airborne images offer the opportunity to recognize features such as road, vegetation, buildings and other kind of infrastructures. The advantage of remote sensing and its applications made it possible to extract damaged, undamaged building and vulnerability assessment of wide urban areas due to a natural disaster. In this paper, we focus on an automatic building detection method which is helpful to optimizing, recognizing, rescuing, recovery and management tasks in the event of a disaster. Objective of this study is to develop techniques for tsunami damaged building extraction, based on very high resolution (VHR) airborne images acquired before and after the 2011 East coastline of Japan among Tohoku area and to carry out a damage assessment of building and vulnerable area mapping. This paper presents a methodology and results of evaluating damaged buildings detection algorithm using an object recognition task based on Mathematical Morphological (MM) operators for Very High Resolution (VHR) remotely sensed airborne images. The proposed approach involves several advanced morphological operators among which an adaptive hit-or-miss transform with varying size and shape of the structuring elements. VHR airborne images consisting of pre and post 2011 Pacific coast of Tohoku earthquake and Tsunami site of the Ishinomaki, Miyagi area in Japan were used. The extracted results of building were compared with ground truth data giving $76 \%$ and $88 \%$ in accuracy before and after the Tsunami event.
\end{abstract}

\section{INTRODUCTION}

With the increase of natural hazards on urban areas in recent years, space borne and airborne remote sensing has been an important tool used for recognizing, rescuing, recovery and managing tasks in the event of a disaster. In the past decade, many kinds of method have developed especially for geometric classification and feature extraction. The VHR remote sensing images offer the opportunity to recognize features such as road, vegetation, buildings and other kind of infrastructures. Automatic extraction of damaged and undamaged man-made structures is a fundamental task in image processing. Among these methods, mathematical morphology has already proved to be effective for many applications in remote sensing (Destival et al., 2009; Heijmans et al., 1998; Lefèvre et al., 2007; Soille et al., 2002, Sun et al., 2008). Classification and feature extraction for remote sensing images from urban area based on morphological transformations and classification of hyper spectral data from urban areas based on extended morphological profiles were presented by Benediktsson et al (Benediktsson, 2003). Similarly, Aaron K. Shackelford et al was investigated a method for automated 2-D building footprint extraction from high-Resolution satellite multispectral imagery (Shackelford, 2004). There are different kinds of hazard area detection algorithms that have been developed by researches using remote sensing applications. However, for most of the above studies, disaster identifications methods still need more improvements.

\section{OBJECTIVES AND METHODOLOGY}

\subsection{Objectives}

The objective of this study is to develop techniques for tsunami damaged building extraction, based on airborne platform based images acquired before and after the 2011 East coastline of Japan among Tohoku area and to carry out a damage assessment of building and vulnerable area mapping. The produce of damage maps are helpful for assist the short and long term reconstructions. Morphological operation of opening, closing with reconstruction, binarization and hit-or-miss transform were applied for image segmentation and building extraction.

\subsection{Mathematical Morphology Operators}

Here the Mathematical Morphology (MM) operators were developed by feature detectors attempts to identify buildings, shadows, roads and other urban features mainly for grey colour images. These vectored profiles were created using morphological opening and closing by reconstruction with different structure elements (SE). 
VHR airborne gray color images of pre and post tsunami event

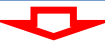

Image registration and mathematical morphology operations

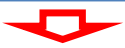

Filter the vectored structuring functions and binary image generation

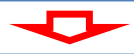

Hit-or-Miss transform

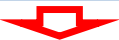

Building extraction and damage estimation

Figure.1 Flow chart of the study

Differential Morphology Profile (DMP) employs a set of image operators to extract and analyse image components based on shape and size of quasi-homogeneous regions in the image. This concept is used to create a feature vector from a single image, $I$ and it is based on the repeat use of the opening and closing operators, which are commonly used in mathematical morphology (Vincent et al., 1993). Opening and closing by reconstruction is obtained following by erosion and dilation under the original image (Pesaresi et al., 2001.). The grey-scale reconstruction $\rho^{* f(p)}$ of image $I$ could be defined as follows. Opening $\gamma$ is defined as the result of erosion followed by the dilation.

$$
\gamma^{*}{ }_{\mathrm{N}} f(p)=\rho^{* f(p)}\left(\varepsilon_{N} f(p)\right)=\operatorname{Re} c\left(\varepsilon_{N} f, f\right)
$$

In a similar fashion, closing $\varphi$ by reconstruction can be defined as

$$
\varphi^{*}{ }_{\mathrm{N}} f(p)=\rho^{* f(p)}\left(\delta_{N} f(p)\right)=\operatorname{Re} c\left(\delta_{N} f, f\right)
$$

Here in the Euclidean transforms assume that flat structuring element that corresponds to the neighbourhood SE= $N_{G}(p)$.The erosion $\varepsilon_{N}$ of the grey level function using the structuring element $N$ is defined by the infimum of the values of the grey level function in the neighbourhood

$$
\left.\varepsilon_{N} f(p)=\left\{\wedge f\left(p^{\prime}\right) \mid p^{\prime}\right) \in N_{G}(p) \cup f(p)\right\}
$$

And the Dilation $\delta_{N}$ is similarly defined by the supremum of the neighboring values and the value of as

$$
\left.\delta_{N} f(p)=\left\{\vee f\left(p^{\prime}\right) \mid p^{\prime}\right) \in N_{G}(p) \cup f(p)\right\}
$$

Opening and closing by reconstruction can be considered as lower-leveling opening and upper-leveling closing operations [13].

\subsection{Generation of binary images}

Selected threshold value $\left(t_{i}\right)$ that defending on each image was applied for pre generated Differential Morphological Profile (DMP) profile images. Binary Image $\left[T_{\left.t_{i}\right]}(f)\right](x)$ serious with different structure elements that contain 0 or 1 pixel value was built up using this threshold.

$$
\left[T_{t_{i}}(f)\right](x)=\left\{\begin{array}{l}
1, \text { if } t_{i} \leq f(x) \\
0, \text { otherwise }
\end{array}\right.
$$

The threshold values were found according to $t_{i} \leq f(x) \leq t_{j}$, in many cases here it assumes threshold value between 140 to 255 and was enough the brighter or darker building roof must be determined empirically classified to pixel value 1 .

\subsection{Hit-or-miss transform for binary case}

The Hit-or-miss transformation, HMT, of a set $\mathrm{x}$ by a composite structuring element $N=\left(N_{F G}, N_{B G}\right)$ is the set of points, $\mathrm{x}$, such that when the origin of $\mathrm{N}$ coincides with $\mathrm{x}, N_{F G}$ fits $f(x)$ while $N_{B G}$ fits $f(x)^{C}$ :

$$
H M T_{N} f(x)=\left\{x \mid\left(N_{F G}\right)_{x} \subseteq f(x),\left(N_{B G}\right)_{x} \subseteq f(x)^{C}\right.
$$

Here we applied (3,9),(5,13) and $(7,17)$ of $N=\left(N_{F G}, N_{B G}\right)$ disk shape structuring elements sets for undamaged buildings extraction which consists of $7,11,15$ structuring elements in MM.

\subsection{Used Data}

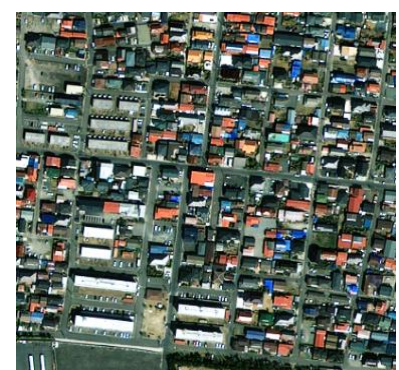

(a)

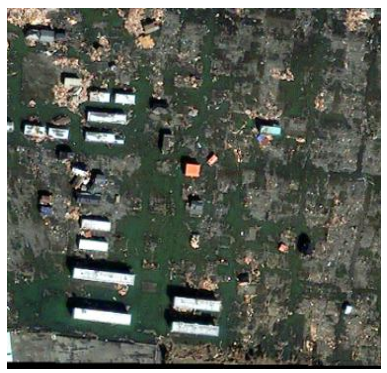

(b)
Figure. 2 The RGB color images show Ishinomaki area before (a) and after (b) the Tsunami event.

The morphological filter theory was designed for a series of gray-level images. The pre generated gray scale airborne images of 2011 Pacific coast Tohoku earthquake and Tsunami site of the Ishinomaki area in Miyagi Prefecture, Japan were used for the study. IDL programming language and ENVI 4.8 commercial software package were used for image processing and classification. Here the figure 2 shows the RGB color images of Tsunami hazardous Ishinomaki City area Miyagi Prefecture, Japan, before and after the 2011 tsunami event. 


\section{BUILDING EXTRACTION}

The maximum DMP response indicates well with a matched SE value that the pixel resides within. There were 3 differential morphological opening profiles that were created using square to $15 \mathrm{~m}$ (step size was equal to $4 \mathrm{~m}$ ). The $\mathrm{SE}$ that less than $7 \mathrm{~m}$ was not reliable for use because they consist of small shadows and rubble of buildings. Because those figures give noise for the classification results, we used SE more than $7 \mathrm{~m}$ to detect remain Buildings. Most of the bright building roofs gave the maximum response to the opening, roads and dark shadows were responded shape morphological elements with square size (s) increasing 7 to the closing.

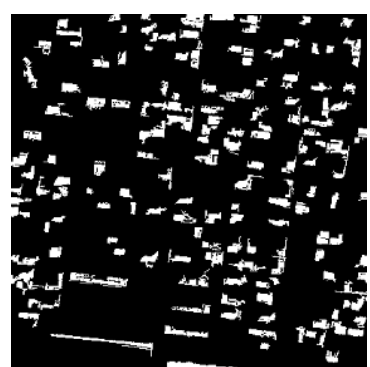

(c) Opening $s=7$

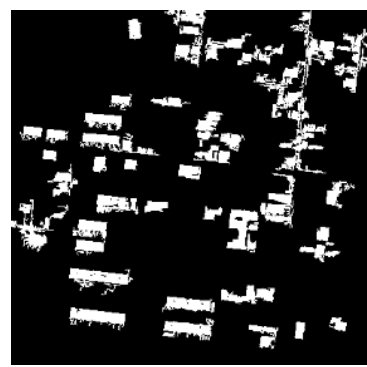

(d) Opening s=11

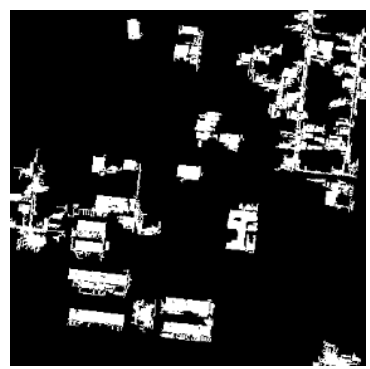

(e) Opening $s=15$

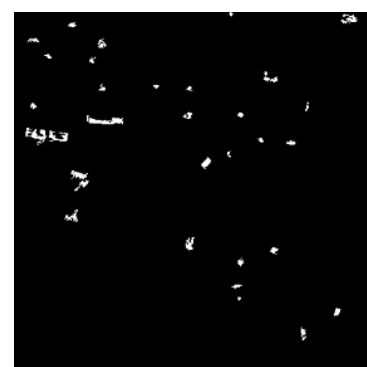

(f) Opening $\quad s=7$

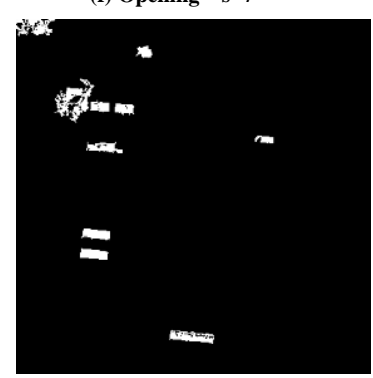

(g) Opening $s=11$

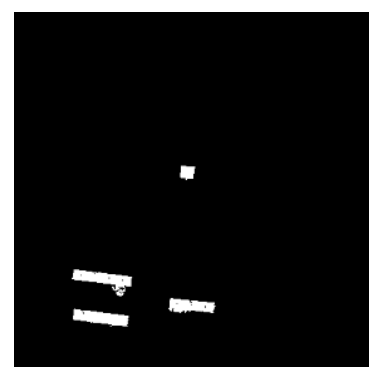

(h) Opening $\mathrm{s}=15$ extract correspond building roofs. The data set included $7 \mathrm{~m} \mathrm{x}$ $7 \mathrm{~m}(3 \mathrm{~m} \times 3 \mathrm{~m}$ foreground and $9 \mathrm{~m} \times 9 \mathrm{~m}$ background), $11 \mathrm{~m}$ $\mathrm{x} 11 \mathrm{~m}(5 \mathrm{~m} \times 5 \mathrm{~m}$ foreground and $13 \mathrm{~m} \times 13 \mathrm{~m}$ background)and $15 \mathrm{mx} 15 \mathrm{~m}(7 \mathrm{~m} \times 7 \mathrm{~m}$ foreground and $17 \mathrm{~m} \times 17 \mathrm{~m}$ background) template windows.

\section{INTERPRETATIONS AND QUALITY ASSESMENT}

The results have shown the usefulness of the proposed method during detection of various types of building, as illustrated by the portions given in Figure 4

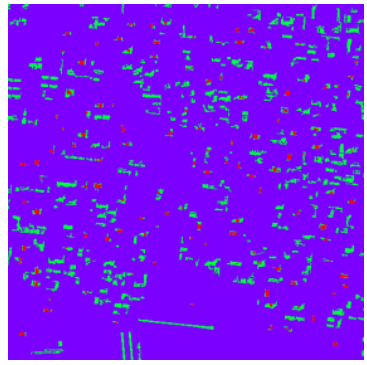

(i)

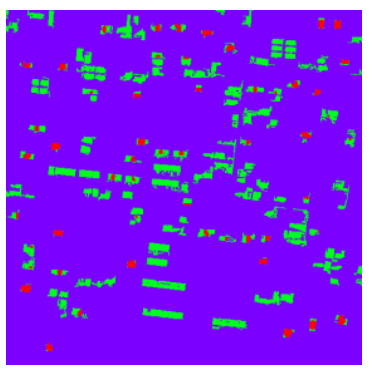

(j)

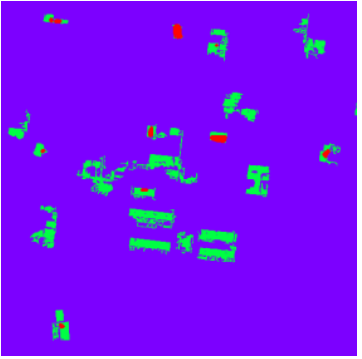

(k)

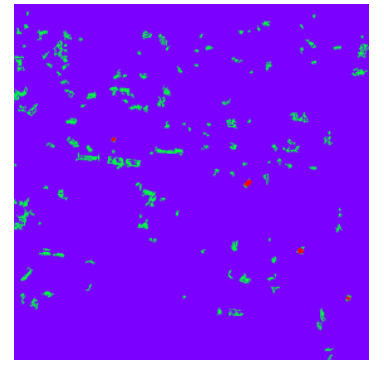

(1)

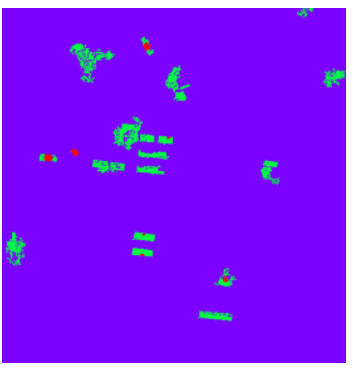

(m)

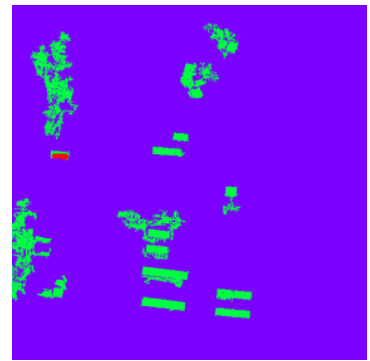

(n)
Figure.3 Images (c) to (e) represent binarized structural decomposition of the pre-event image differential morphological profile and (f) to (h) represents post event differential morphological profile. The images have been visually enhanced. The derivative has been calculated relative to a series generated by 3 iterations of the elementary SE with 7$15 \mathrm{~m}$ rectangular shape roofs. Derivative of the opening profile with $\mathrm{s}=7(\mathrm{c}, \mathrm{f}), 11(\mathrm{~d}, \mathrm{~g})$ and $15(\mathrm{e}, \mathrm{h})$ are shown above respectively

Simple image binarization threshold was applied to each DMP profiles for noise reduction and avoid misclassification. Figure 3 shows the binarized images of both pre and past event differential morphological opening profiles. The segmentation threshold value was set to 70 pixels and then the template matching based on Hit or miss transform method was applied to
Figure.4 Result of the building extraction according to approached method. Identified buildings are shown in red color, (i),(l) pre and post event building roofs corresponded to $\mathrm{SE}=7$, (j),(m) pre and post event building roofs corresponded to $\mathrm{SE}=11$ and $(\mathrm{k}),(\mathrm{n})$ show the pre and post event building roofs corresponded to $\mathrm{SE}=15$ respectively.

The quality of the results was assessed with exist GIS data (pre event) and visual inspection following manually labeling (post event) as ground truth. Although this result appeared to be slightly high, the confidence measures produced by the suggested a reliability of pre and post event gives $76.41 \%$ and $88.26 \%$ in object based accuracy. For the applied area, the 
method detected 1225 buildings among 1592 before the tsunami event and 425 buildings among 482 after the event. The error extraction of building structure could be due to over fitting of the decision surface to the data.

\section{CONCLUTIONS}

We applied a method for extraction of urban structures and hazard estimation using VHR airborne images. This method is adapted to pre and post event gray color images and do not require any kind of ancillary data to be performed. The first step was to segment structural information using morphological opening and closing by reconstruction operators. There for the pre-generated gray level airborne images of pre and postearthquake and tsunami event in Ishinomaki area in Miyagi prefecture were applied to the morphological operators. The shadows of the buildings were masked and removed using their low spectral values. This work is a further extension of our previous study by introducing binary images and hit-or-miss transform. The segmented images were applied to hit-or-miss transform to extract the building roofs. Proposed combinations of building roofs reflectance value and shape increased the probability of building extraction. The candidate area contained various kinds of roofs with different color, shape and size. However, there were some building structures that are complex, hence these sometimes combine together to be classified as a one building. Further work is required to increase the accuracy of building detection and determine if damage ratio of the structure can be estimated.

\section{Acknowledgements}

We would like to thank for access airborne imagery and GIS data, provided by Geospatial Information Authority of Japan (GSI), Ministry of Land, Infrastructure, Transport and Tourism. The authors also acknowledge to Gareth Wyvill and Duminda Welikanna, Kyoto University, for knowledge sharing and facilitating.

\section{References}

Aytekin O., Ulusoy I., Abacioglu E.Z., and Gokcay E., 2009, "Building detection in high resolution remotely sensed images based on morphological operators", Recent Advances in Space Technologies, RAST '09, pp.376-379.

Benediktsson J.A., Pesaresi M., and Arnason K., 2003, "Classification and Feature Extraction for Remote Sensing Images From Urban Areas Based on Morphological Transformations", IEEE Transactions on Geoscience and Remote Sensing, VOL. 41, NO. 9, pp. 1940-1949.

Heijmans H.J.A.M, and Roerdink B. T. M., 1998, "From connected operators to leveling" in Mathematical Morphology and Its Applications to Image and Signal Processing, Kluwer Academic Publishers, Dordrecht, pp. 191-198.

Lefèvre S., Weber J., and Sheeren D., 2007, "Automatic Building Extraction in VHR Images Using Advanced Morphological Operators", IEEE/ISPRS Joint Workshop on Remote Sensing and Data Fusion over Urban Areas (URBAN), Paris.
Derivaux S., Lefèvre S., Wemmert C., and Korczak J., 2006, "Watershed segmentation of remotely sensed images based on a supervised fuzzy pixel classification", IEEE International Geosciences and Remote Sensing Symposium, Denver, USA, pp. 3712-3715.

Destival I., 2009, "Mathematical morphology applied to remote sensing”, Acta Astronautica, vol. 13, no. 6/7, pp. 371-385.

Pesaresi M., and Benediktsson J.A., 2001, "A New Approach for the Morphological Segmentation of High-Resolution Satellite Imagery", IEEE International Geosciences And Remote Sensing Symposium, Vol.39, No. 2, pp. 309-319.

Shackelford A.K, and Davis C.H., 2004, "Automated 2-D Building Footprint Extraction from High-Resolution Satellite Multispectral Imagery A Hierarchical Fuzzy Classification Approach", IEEE International Geosciences And Remote Sensing Symposium, pp.1996-1999.

Soille.P, and Pesaresi M., 2002 "Advances in mathematical morphology applied to geoscience and remote sensing", IEEE Transactions on Geoscience and Remote Sensing, vol. 40, No.9, pp. 2042-2055.

Sun X., Fu K., Long H., Yanfeng H., CaiL., and Wang H.,2008, "Contextual Models for Automatic Building Extraction in High Resolution Remote Sensing Image Using Object-Based Boosting Method", Geoscience and Remote Sensing Symposium, IGARSS .

Vincent L., 1993, "Morphological area openings and closings for gray scale images", Shape and Picture: Math. Description of shape in gray level images, NATO, p.p. 197-208. 